\title{
A Case of Rhizomelic Chondrodysplasia Punctata in Newborn
}

\author{
Nalan Karabayır, ${ }^{1}$ Gonca Keskindemirci, ${ }^{1}$ Erdal Adal, ${ }^{1}$ and Orhan Korkmaz ${ }^{2}$ \\ ${ }^{1}$ Pediatrics Department, Bakırköy Maternity and Children Education and Research Hospital, \\ Kartaltepe mah Aksoy sok. Petrol Sitesi 6/11 Bakırköy, Istanbul, Turkey \\ ${ }^{2}$ Radiology Department, Bakırköy Maternity and Children Education and Research Hospital, Turkey \\ Correspondence should be addressed to Nalan Karabayır; nalankarabayir@hotmail.com
}

Received 13 October 2013; Revised 26 January 2014; Accepted 28 January 2014; Published 9 March 2014

Academic Editor: Simon Ching-Shun Kao

Copyright (c) 2014 Nalan Karabayır et al. This is an open access article distributed under the Creative Commons Attribution License, which permits unrestricted use, distribution, and reproduction in any medium, provided the original work is properly cited.

Rhizomelic chondrodysplasia punctate (RCDP) is a rare autosomal recessive peroxisomal disease. The main features of the disease are shortening of the proximal long bones, punctate calcifications located in the epiphyses of long bones and in soft tissues around joints and vertebral column, vertebral clefting, dysmorphic face, and severe growth retardation, whereas cervical spinal stenosis may also rarely be present. Imaging of the brain and spinal cord in patients with this disorder may aid prognosis and guide management decisions. We report the newborn diagnosed as CDP with cervical stenosis. Our aim is to discuss current knowledge on etiopathogenesis as well as radiological and clinical symptoms of diseases associated with CDP.

\section{Introduction}

Rhizomelic chondrodysplasia punctata (RCDP) is a rare disorder of peroxisomal metabolism, with an estimated incidence $1: 100.000$. There are 3 genetic subtypes. RCDP type 1 (OMIM 215100), caused by mutations in the PEX7 gene, is the most common type. RCDP type 2 (OMIM 222765) and 3 (OMIM 600121) are single enzyme deficiencies in the plasmalogen biosynthesis pathway. RCP type 2 arises secondary to mutations in the acyl-CoA:dihydroxyacetone phosphate acyltransferase (DHAPAT) gene, and RCP type 3 arises from mutations in the alkyl-dihydroxyacetone phosphate synthase (ADAPS) gene $[1,2]$. The main features of the disease are shortening of the proximal long bones, punctate calcifications in the metaphysis and epiphysis of long bones and the thoracic and lumbar vertebrae, dysmorphic face, and severe growth retardation. Cervical stenosis is very rarely reported in rhizomelic CDP cases $[1,3]$. Because of underlying vertebral abnormalities, spinal stenosis, often seen together with brachytelephalangic chondrodysplasia punctata, can cause progressive neurological findings. Here, we report a case of RCDP with cervical spinal stenosis in newborn.

\section{Case}

The term infant was admitted to the neonatology department because of its atypical facial appearance and extremity anomalies at the 2 nd hour of her life. The female infant was born at 38 weeks of gestation from the fourth pregnancy of a healthy 22-year-old mother and a 30-year-old related father. The mother was under routine prenatal follow-up during pregnancy. She did not have any chronic disease and there was no history of exposure to any known embryopathic agents and, in particular, no warfarin therapy or alcohol use had been given. Prenatal ultrasonographic assessments reported proximal limb shortening. The mother had two miscarriages, as well as a baby with skeletal abnormalities who was aborted at 22 gestational weeks and a healthy male child. On the physical examination, BW was $3200 \mathrm{gm}$ (5075 percentiles), height was $50 \mathrm{~cm}$ (75-90 percentiles), head circumference was $35 \mathrm{~cm}$ (75-90 percentiles), the neck was deviated to the right, and there was a depressed nasal bridge and a highly arched palate. There was shortness of the upper extremities and flexion contractures in all extremities. The infant's systemic examination was otherwise normal. In the skeletal survey performed, there were proximal shortness, 


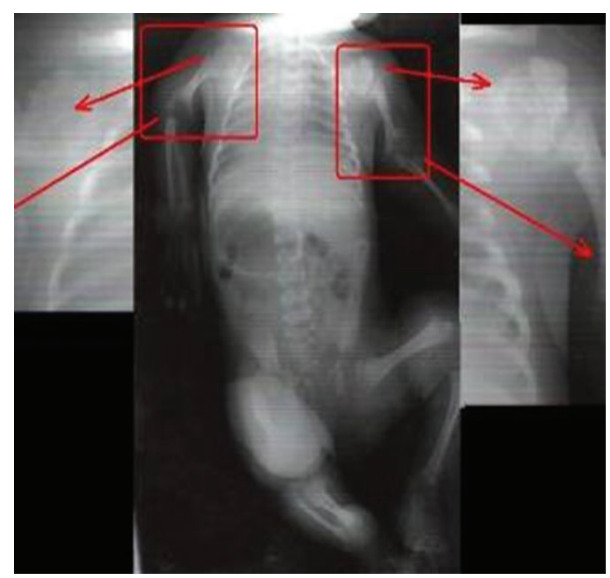

FIgURE 1: Proximal shortness, thick and short diaphyses, large and irregular metaphyses, and punctate calcifications in the epiphyses in the long bones and coronal clefts not included in the vertebral bodies.

thick and short diaphyses, and large and irregular metaphyses in the long bones and normal fingers. The radiological findings of the patient were compatible with CDP with punctate calcifications in the epiphyses and coronal clefts in the vertebral bodies (Figure 1). Complete blood count, biochemical parameters, and abdominal ultrasonography were all normal. Secundum ASD and thin PDA were detected on echocardiography. Cavum vergae and minimal dilatation in the right ventricle were observed on cranial ultrasonography. On the cranial MR investigation, both lateral ventricles and the third ventricle were found to be larger than normal and there was atrophy in the left temporal lobe. On the cervical spinal MRI, spinal stenosis was detected at C4-5, C5-6, and C6-7 levels (Figure 2). Bilateral nuclear cataract was seen on the ophthalmological examination. Her karyotype test was normal $(46, \mathrm{XX})$. Blood amino acid and urinary organic acid levels were unremarkable, and anti-DNA and ANA tests of her mother revealed negative results. Further biochemical studies showed high phytanic acid (9.2; $n<5.28 \mu \mathrm{mol} / \mathrm{L})$ and low plasmalogen (3.2; $n>6.6)$ levels. RCDP1 was diagnosed based on clinical, biochemical, and radiological criteria. Parents were informed about this disease, and genetic counseling was given. The case is now 2 months of age, weighing $4800 \mathrm{gr}$, and is fed orally. She was operated at the age of 1 month for bilateral cataract. She has been in followup for spinal stenosis and probable nutritional problems. Echocardiography was planned to monitor the size of heart defect after 6 months.

\section{Discussion}

Chondrodysplasia punctata (CDP) is a rarely occurring skeletal dysplasia characterized by stippled, punctuate calcifications around joints and within cartilages [1]. CDP is associated with a number of disorders, including inborn errors of metabolism, involving peroxisomal and cholesterol pathways, embryopathy, and chromosomal abnormalities [2-7]. Several classification systems of the different types

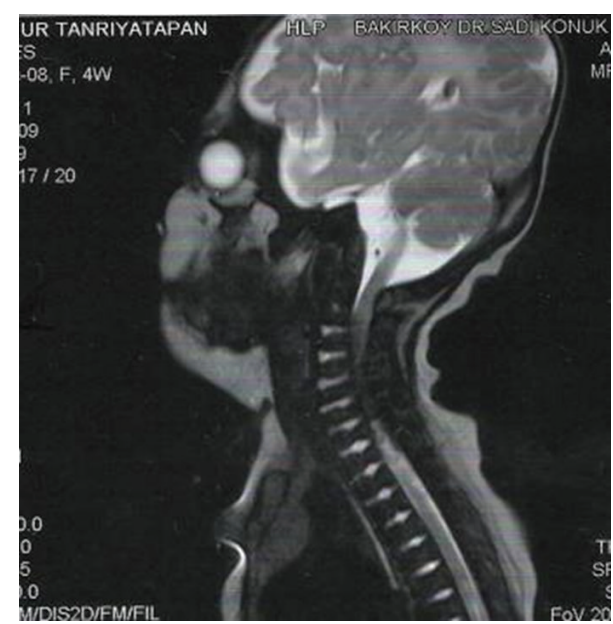

FIGURE 2: On the cervical spinal MRI, spinal stenosis at C4-5, C5-6, and C6-7 levels.

of CDP have been suggested earlier. More recently, the biochemical and molecular basis of a number of CDP syndromes has recently been elucidated and a new aetiological classification has emerged [2]. Rhizomelic chondrodysplasia punctata is a disorder caused by abnormal peroxisomal function which can be mediated both through disorders of biosynthesis, for example, peroxisomal assembly (RDCP1), and by single enzyme defects, affecting plasmalogen synthesis (RCDP2, RCDP3). Clinically, RCDP1, RCDP2, and RCDP3 are characterized by rhizomelic shortening, mainly affecting the humerus, facial dysmorphism, seizures, cataracts, and joint contractures. Growth and development are severely restricted. Life expectancy is considerably reduced $[1,2$, 8]. Pathognomonic finding for RCDP is a reduced level of plasmalogens with normal very long chain fatty acids (VLCFA) [1]. The following situations should be considered in the differential diagnosis of CDP: peroxisomal diseases (Zellweger Syndrome, adrenoleukodystrophy, and infantile Refsum disease), maternal disease, and exposure to warfarin, Smith-Lemli-Opitz Syndrome, and foetal alcohol syndrome $[2,9,10]$. There was no history of maternal drug or alcohol use and no symptoms or positive laboratory test that indicated autoimmunological disease in the mother. Because our case had high phytanic acid level and autosomal recessive inheritance, she was diagnosed as RCDP1.

Spinal stenosis is a frequent sign of bone dysplasia, while it is rarely reported in rhizomelic CDP cases [11-13]. Cervical spinal stenosis, which in some cases leads to cord compression and myelopathy, has been described in chondrodysplasia punctate of rhizomelic, brachytelephalangic, and ConradiHunermann types [13]. Because patients with RCDP often demonstrate upper and lower extremity spasticity in the absence of spinal cord involvement, diagnosis of cervical spinal stenosis secondary to RCDP may be difficult. In addition, it is sometimes hard to establish the clinical finding of spasticity because multiple joints have limited range of motion [14]. Also, a recent study found an association between overall disease severity and the presence of abnormalities on brain magnetic resonance imaging [15]. Because 
neuroimaging can provide prognostic information, it is important to look for cervical stenosis in these patients. These patients may be screened with conventional radiographs of the cervical spine and cases with radiographs suggestive of spinal stenosis and physical examinations compatible with myelopathy may be further evaluated with MRI when clinically indicated [14]. In our case, cervical stenosis was detected on the spinal MRI investigations performed for this purpose. But our patient who had cervical stenosis still has no neurological findings.

Spasticity, psychomotor retardation, growth retardation, seizures, thermoregulatory instability, feeding difficulty, recurrent otitis media, and pneumonia have been reported in CDP cases [11]. Our case was still fed orally in spite of the feeding difficulty, and her weight was $4800 \mathrm{gm}(50-75 \mathrm{p})$ at two months. So, our patient was described as mildly affected.

Prenatal diagnosis of RCDP is possible from the first trimester onwards by demonstration of peroxisomal dysfunction in cultured chorionic villous or amniotic fluid cells [3]. Genetic counseling was given to parents of our case.

In conclusion, cervical spinal stenosis is a rare anomaly in rhizomelic chondrodysplasia punctata cases, which may cause neurological findings. Imaging of the brain and spinal cord in patients with this disorder may aid prognosis and guide management decisions.

\section{Conflict of Interests}

The authors declare that there is no conflict of interests regarding the publication of this paper.

\section{References}

[1] A. M. Bams-Mengerink, J. H. Koelman, H. Waterham, P. G. Barth, and B. T. Poll-The, "The neurology of rhizomelic chondrodysplasia punctata," Orphanet Journal of Rare Diseases, vol. 8, no. 1, article 174, 2013.

[2] M. D. Irving, L. S. Chitty, S. Mansour, and C. M. Hall, "Chondrodysplasia punctata: a clinical diagnostic and radiological review," Clinical Dysmorphology, vol. 17, no. 4, pp. 229-241, 2008.

[3] C. T. Yalin, I. K. Bayrak, M. Danaci, and L. Incesu, "Case report: rhizomelic chondrodysplasia punctata and foramen magnum stenosis in a newborn," Turkish Journal of Diagnostic and Interventional Radiology, vol. 9, no. 1, pp. 100-103, 2003.

[4] S. C. Morrison, "Punctate epiphyses associated with Turner syndrome," Pediatric Radiology, vol. 29, no. 6, pp. 478-480, 1999.

[5] A. Leicher-Duber, R. Schumacher, and J. Spranger, "Stippled epiphyses in fetal alcohol syndrome," Pediatric Radiology, vol. 20, no. 5, pp. 369-370, 1990.

[6] J.-L. D. Alessandri, D. Ramful, and F. Cuillier, "Binder phenotype and brachytelephalangic chondrodysplasia punctata secondary to maternal vitamin K deficiency," Clinical Dysmorphology, vol. 19, no. 2, pp. 85-87, 2010.

[7] A. K. Poznanski, "Punctate epiphyses: a radiological sign not a disease," Pediatric Radiology, vol. 24, no. 6, pp. 418-424, 1994.

[8] A. L. White, P. Modaff, F. Holland-Morris, and R. M. Pauli, "Natural history of rhizomelic chondrodysplasia punctata," American Journal of Medical Genetics, vol. 118, no. 4, pp. 332-342, 2003.
[9] A. L. Shanske, L. Bernstein, and R. Herzog, "Chondrodysplasia punctata and maternal autoimmune disease: a new case and review of the literature," Pediatrics, vol. 120, no. 2, pp. e436-e441, 2007.

[10] I. Singh, G. H. Johnson, and F. R. Brown III, "Peroxisomal disorders. Biochemical and clinical diagnostic considerations," American Journal of Diseases of Children, vol. 142, no. 12, pp. 1297-1301, 1988.

[11] P. Violas, B. Fraisse, M. Chapuis, and H. Bracq, "Cervical spine stenosis in chondrodysplasia punctata," Journal of Pediatric Orthopaedics B, vol. 16, no. 6, pp. 443-445, 2007.

[12] T. E. Herman, B. C. P. Lee, and W. H. McAlister, "Brachytelephalangic chondrodysplasia punctata with marked cervical stenosis and cord compression: report of two cases," Pediatric Radiology, vol. 32, no. 6, pp. 452-456, 2002.

[13] E. Jurkiewicz, B. Marcinska, J. Bothur-Nowacka, and A. Dobrzanska, "Clinical and punctate and review of available literature," Polish Journal of Radiology, vol. 78, no. 2, pp. 57-64, 2013.

[14] J. Khanna, N. E. Braverman, D. Valle, and P. D. Sponseller, "Cervical stenosis secondary to rhizomelic chondrodysplasia punctata: brief clinical report," American Journal of Medical Genetics, vol. 99, no. 1, pp. 63-66, 2001.

[15] A. M. Bams-Mengerink, C. B. L. M. Majoie, M. Duran et al., "MRI of the brain and cervical spinal cord in rhizomelic chondrodysplasia punctata," Neurology, vol. 66, no. 6, pp. 798-803, 2006. 


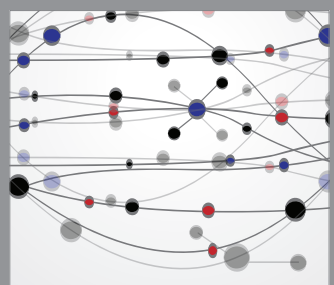

The Scientific World Journal
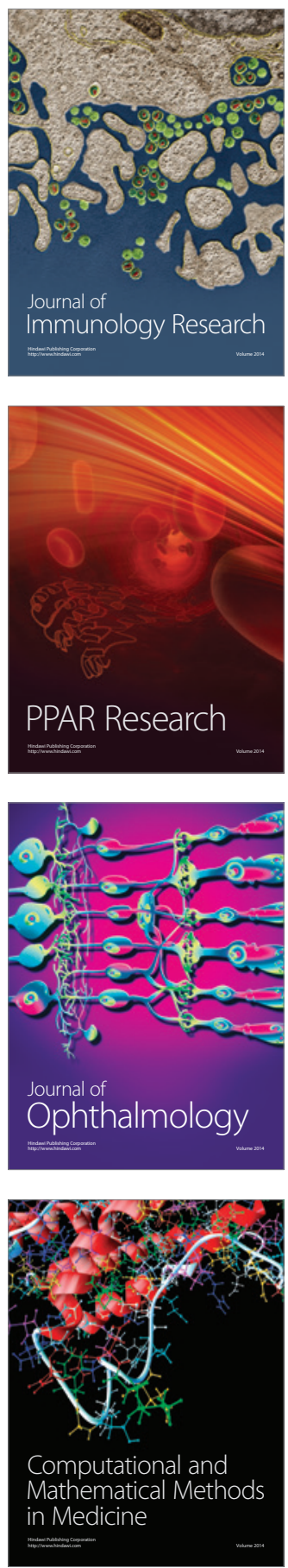

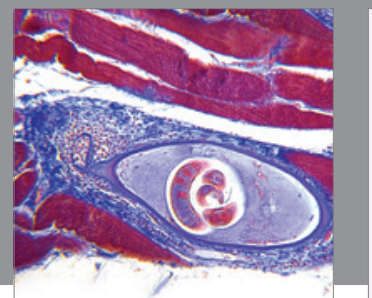

Gastroenterology

Research and Practice
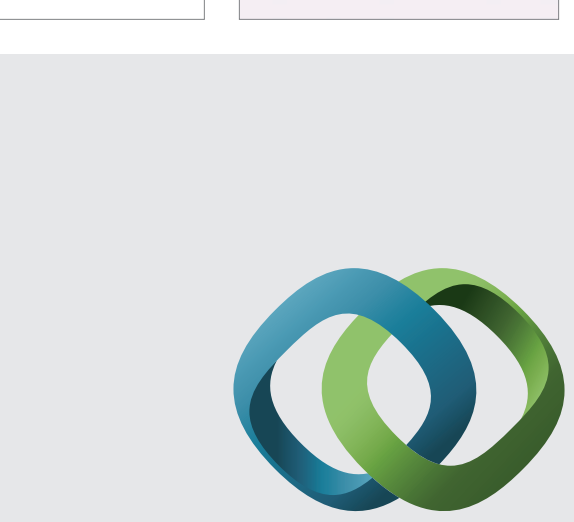

\section{Hindawi}

Submit your manuscripts at

http://www.hindawi.com
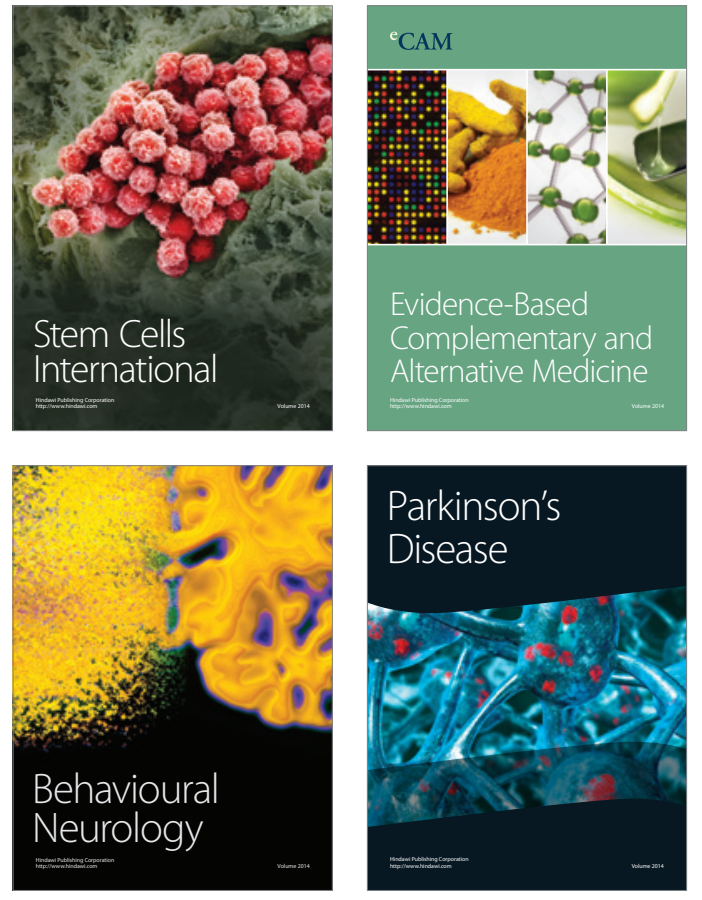
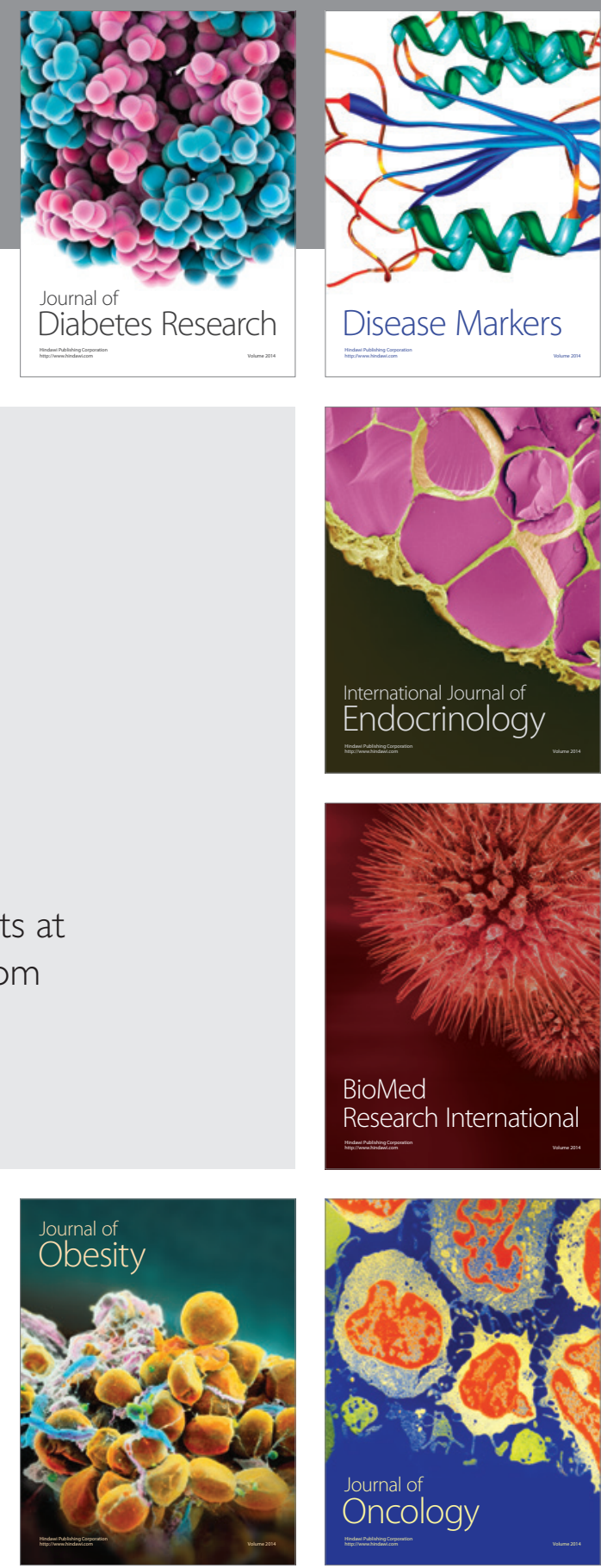

Disease Markers
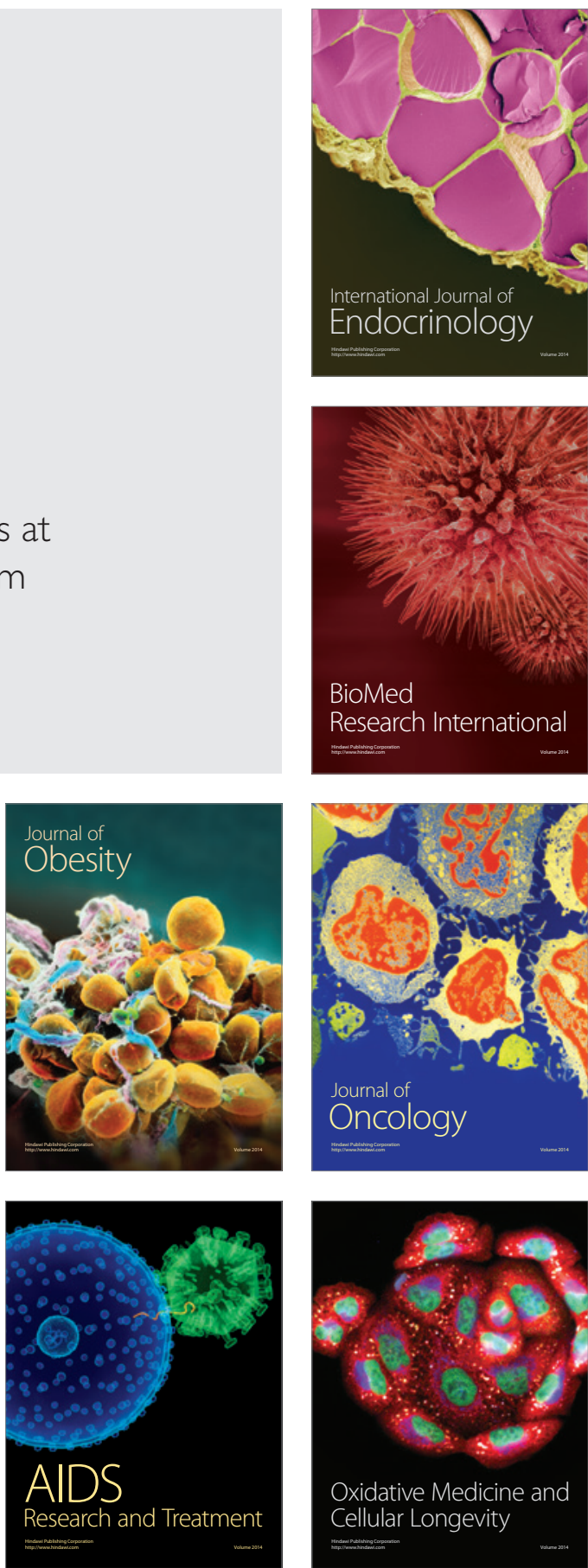